\title{
On-chip thermal testing using MOSFETs in weak
}

\section{inversion}

$\underline{\text { Authors: Ferran Reverter }}{ }^{\mathrm{a}}$ and Josep Altet ${ }^{\mathrm{b}}$

Address: $\quad$ Department of Electronic Engineering Universitat Politècnica de Catalunya (UPC) - BarcelonaTech

${ }^{a}$ C/ Esteve Terradas 7, C4, 08860 Castelldefels (Barcelona), Spain

${ }^{\mathrm{b}} \mathrm{C} /$ Jordi Girona 1-3, Campus Nord, C4, 08034 Barcelona, Spain

Corresponding author: $\quad$ Ferran Reverter, ferran.reverter@upc.edu

Phone: +34 934137076

Telefax: +34 934137007 
Abstract-This paper analyses the feasibility of using metal-oxide-semiconductor field-effect transistors (MOSFET) operating in weak inversion as temperature sensors for on-chip thermal testing applications. MOSFETs in weak inversion are theoretically analyzed so as to know how their sensitivity to temperature depends on both dimensions and bias current. Theoretical predictions are then compared with simulations and experimental data resulting from MOSFETs fabricated in a commercial $0.35 \mu \mathrm{m}$ CMOS technology. MOSFETs are experimentally subjected to changes of temperature generated by either a heating chamber or an on-chip power dissipating device. The performance of MOSFETs in weak inversion is also compared with that in strong inversion and with that of parasitic bipolar junction transistors (BJT). In the context of on-chip thermal testing, MOSFETs in weak inversion offer advantages in terms of layout area, linearity, current consumption and spread of the sensitivity to temperature due to process variations.

Keywords: MOSFET sensor; Subthreshold operation; Temperature sensor; Thermal testing; Weak inversion. 


\section{INTRODUCTION}

Thermal testing and characterization of integrated circuits (IC), e.g. digital circuits $[1,2]$ and analogue radio frequency $(\mathrm{RF})$ circuits $[3,4]$, through temperature sensors embedded into the same chip has been proved useful in the last years. The temperature sensor is placed close to the circuit under test (CUT) and measures on-chip thermal variations caused by the power dissipated by the CUT with the aim of extracting information, for example: (i) the efficiency (i.e. the DC power dissipated by the load divided by the DC power delivered by the DC source) of a RF power amplifier operating at $2 \mathrm{GHz}$ [3], and (ii) the frequency response and the central frequency (at $830 \mathrm{MHz}$ ) of a RF low-noise amplifier [4]. In comparison with classical IC test techniques based on electrical measurements [5-7], thermal testing offers two main advantages for RF-ICs: the CUT is not electrically loaded, and measurements are

performed at DC [3] or at low frequency $[4,8]$, which reduces the complexity and cost of the test.

On-chip thermal testing of RF-CUTs has been performed in $[3,4]$ using the well-known temperature dependence (i.e. $-2 \mathrm{mV} / \mathrm{K}$ ) of the base-emitter voltage of a parasitic BJT fabricated in CMOS technology [9]. However, the comparative analysis carried out in [10] shows that MOSFET-based temperature sensors (operating in strong inversion) offer attractive advantages for such applications: (i) fully compatibility with the fabrication process, (ii) less layout area (say, ten times less) required around the CUT, and (iii) more sensitivity (up to three times more) to on-chip thermal variations caused by the CUT. The main drawback of MOSFETs is that their sensitivity to temperature is more susceptible (say, four times more) to IC manufacturing process variations [10].

This paper goes a step further in the analysis of MOSFETs as temperature sensors for onchip thermal testing applications. The approach proposed in [10] is applied herein to analyze the feasibility of using MOSFETs in weak inversion, instead of strong inversion, as 
temperature sensors for such applications. Although MOSFETs in weak inversion have been already proposed as low-power temperature sensors $[2,11,12]$, no comparative analysis (with respect to BJTs and MOSFETs in strong inversion) has been carried out in the context of onchip thermal testing. After explaining the IC thermal testing technique behind the proposed temperature sensor, this paper provides theoretical models, simulations and experimental results of MOSFETs operating in weak inversion, and then it compares these results with those reported in [10] with the purpose of extracting the advantages and drawbacks of each sensing transistor.

\section{ON-CHIP THERMAL TESTING TECHNIQUE}

The temperature sensor analyzed herein is mainly intended for the on-chip thermal characterization of RF circuits using the heterodyne technique [4]. This technique operates as follows: two tones of high frequency $f_{1}$ and $f_{2}=f_{1}+\Delta f$ ( $\Delta f$ being, for instance, $1 \mathrm{kHz}$ ) are applied to the input of the RF-CUT (see Fig. 1a) and, as a consequence of the frequency mixing generated by Joule effect [13], the RF-CUT dissipates power at low frequency (i.e. at $\Delta f$ ) with information about the performance at high frequency (i.e. at $f_{1}$ ). Accordingly, the frequency response of a RF-CUT can be achieved by monitoring the amplitude of the power dissipated at $\Delta f$ for different values of $f_{1}[4]$. This power dissipated at $\Delta f$ generates an on-chip temperature signal at the same frequency that can be measured by a nearby temperature sensor $\left(T_{1}\right.$ in Fig. 1a) that is thermally coupled to the CUT through the semiconductor substrate; note that the low-pass filter response of the thermal coupling filters out other spectral components of the dissipated power (such as those at $f_{1}, f_{2}, f_{1}+f_{2}, \ldots$ ). The temperature sensor is then proposed to be connected to an amplifying band-pass filter (BPF) whose passband is centered at $\Delta f$ so as to limit the bandwidth (and, hence, the noise) around the frequency of interest [8]. The BPF also rejects slow variations of the ambient temperature affecting the IC, and slow variations of on-chip temperature generated by the DC bias of the 
CUT or by any other heating source.

The simplest temperature sensor that can be employed in Fig. 1a is a diode-connected MOSFET biased with a constant current source $\left(I_{\mathrm{B}}\right)$, as shown in Fig. $1 \mathrm{~b}[8,10]$. In this circuit, the MOSFET carries out a small-signal temperature-to-voltage conversion at low frequency (i.e. at $\Delta f$ ) with the aim of extracting high-frequency information of the RF-CUT. Although the DC operating point of that circuit is quite susceptible to process variations, this is not a major concern since the DC level (and, hence, the corresponding absolute value of temperature) will be blocked out by the ensuing BPF shown in Fig. 1a. What it really matters here is the measurement of the temperature change at $\Delta f$ generated by the RF-CUT when the heterodyne technique is applied. For this reason, next sections mainly focus on the ability of the sensor to detect changes of temperature (i.e. the sensitivity to temperature) rather than the accuracy. The amplitude of the temperature change to be detected depends on several factors (such as the operating frequency, the dissipated power, and the distance between the CUT and the temperature sensor), but values around tenths [8] or a few units of Kelvin [13] are expected. In the literature, we can find other thermal applications interested in measuring changes of temperature rather than the absolute value of temperature. For instance, the measurement of the amplitude (and also the phase shift) of temperature oscillations at a given frequency generated by the AC power dissipated by a heating element is used to determine thermal properties of fluids [14] and to monitor biofilm dynamics [15].

\section{THEORETICAL ANALYSIS}

This section analyses the same MOSFET-based temperature sensor proposed in [10] (i.e. a diode-connected n-type MOSFET biased with $I_{\mathrm{B}}$, as shown in Fig. 1b) but in weak inversion (or subthreshold) operation. For the application of interest described in Section II, the current source $I_{\mathrm{B}}$ will be far from and, hence, not affected by the power dissipated by the CUT and, for this reason, $I_{\mathrm{B}}$ is assumed to be constant in the following analysis. Of course, $I_{\mathrm{B}}$ could be 
affected by changes of ambient temperature, but such effects are not expected to be critical here whenever the IC test is faster than the temperature changes affecting $I_{\mathrm{B}}$.

In weak inversion (i.e. $V_{\mathrm{GS}}<V_{\mathrm{TH}}$ ) and in saturation (i.e. $V_{\mathrm{DS}}>5 U_{\mathrm{T}}$ ), the current-voltage characteristic of a MOSFET can be modeled as follows [16]

$$
I_{\mathrm{D}}=\mu C_{\mathrm{OX}} \frac{W}{L}(\eta-1) U_{\mathrm{T}}^{2} \exp \left(\frac{V_{\mathrm{GS}}-V_{\mathrm{TH}}}{\eta U_{\mathrm{T}}}\right),
$$

where $I_{\mathrm{D}}$ is the drain current, $V_{\mathrm{GS}}$ is the gate-source voltage, $V_{\mathrm{DS}}$ is the drain-source voltage, $W$ is the channel width, $L$ is the channel length, $V_{\mathrm{TH}}$ is the threshold voltage, $\mu$ is the carriers mobility, $U_{\mathrm{T}}$ is the thermal voltage, $\eta$ is a subthreshold slope factor, $C_{\mathrm{OX}}\left(=\varepsilon_{\mathrm{OX}} / t_{\mathrm{OX}}\right)$ is the gate oxide capacitance per unit area, $\varepsilon_{\mathrm{OX}}$ is the gate oxide permittivity, and $t_{\mathrm{OX}}$ is the gate oxide thickness. Unlike what happens in strong inversion where the current-voltage characteristic has a quadratic relationship [10], in weak inversion the relationship is exponential, as in BJTs; this is because in weak inversion the channel current flows by diffusion [17].

According to [18], three parameters involved in (1) depend on temperature:

$$
\begin{gathered}
U_{\mathrm{T}}(T)=\frac{k T}{q} \\
\mu(T)=\mu_{0}\left(\frac{T}{T_{0}}\right)^{\alpha} \\
V_{\mathrm{TH}}(T)=V_{\mathrm{TH} 0}+\beta\left(T-T_{0}\right),
\end{gathered}
$$

where $T$ is the absolute temperature, $T_{0}$ is a reference temperature (e.g. $300 \mathrm{~K}$ ), $k$ is the Boltzmann constant $\left(1.38066 \cdot 10^{-23} \mathrm{~J} / \mathrm{K}\right), q$ is the elementary charge $\left(1.60218 \cdot 10^{-19} \mathrm{C}\right), \mu_{0}$ is the carriers mobility at $T_{0}, \alpha$ is a constant generally between -1.5 and $-2, V_{\mathrm{TH} 0}$ is the threshold voltage at $T_{0}$, and $\beta$ is a negative temperature coefficient; the value of such parameters for the 
CMOS technology applied herein is reported in Table I [19]. Note that $\alpha$ and $\beta$ are negative, so both $\mu$ and $V_{\mathrm{TH}}$ decrease with temperature.

For the circuit shown in Fig. $1 \mathrm{~b}$ where the MOSFET is biased with $I_{\mathrm{D}}=I_{\mathrm{B}}$, the output voltage (which is equal to $V_{\mathrm{GS}}$ ) can be expressed, from (1), as

$$
V_{\mathrm{o}}=\eta U_{\mathrm{T}} \ln \left(\frac{I_{\mathrm{B}}}{\mu C_{\mathrm{OX}} \frac{W}{L}(\eta-1) U_{\mathrm{T}}^{2}}\right)+V_{\mathrm{TH}} .
$$

Replacing now (2), (3) and (4) in (5) yields the temperature dependence of $V_{\mathrm{o}}$

$$
V_{\mathrm{o}}(T)=V_{\mathrm{TH} 0}-\beta T_{0}+T(\beta+\gamma)+(\alpha+2) \eta U_{\mathrm{T}} \ln \frac{T_{0}}{T},
$$

where

$$
\gamma=\eta \frac{k}{q} \ln \left(\frac{I_{\mathrm{B}}}{\mu_{0} C_{\mathrm{OX}} \frac{W}{L}(\eta-1) U_{\mathrm{T} 0}^{2}}\right),
$$

$U_{\mathrm{T} 0}$ being the thermal voltage at $T_{0}$ (i.e. $26 \mathrm{mV}$ ). Assuming $\left(T_{0} / T\right) \approx 1$, the logarithmic function involved in (6) can be replaced by its first-order Taylor-series approximation, i.e. $\ln \left(T_{0} / T\right) \approx T_{0} / T-1$. Consequently, Eq. (6) can be simplified to the following first-order temperature-dependent equation

$$
V_{\mathrm{o}}(T) \approx V_{\mathrm{TH} 0}-\beta T_{0}+(\alpha+2) \eta U_{\mathrm{T} 0}+T\left(\beta+\gamma-(\alpha+2) \eta \frac{k}{q}\right),
$$

whose sensitivity to temperature is

$$
S_{\mathrm{T}}=\frac{\partial V_{\mathrm{o}}}{\partial T}=\beta+\gamma-(\alpha+2) \eta \frac{k}{q}
$$

Taking into account that $k / q$ has a low value (i.e. $86 \mu \mathrm{V} / \mathrm{K}$ ) and $\alpha$ is close to -2 , the last term in (9) turns to be much smaller than the others and, therefore, $S_{\mathrm{T}}$ can be approximated to 


$$
S_{\mathrm{T}} \approx \beta+\gamma
$$

A MOSFET operating in weak inversion requires a high $W / L$ and a low $I_{\mathrm{B}}[20,21]$, so the term inside the logarithm in (7) is expected to be smaller than one and, hence, $\gamma$ will be smaller than zero. Therefore, both $\beta$ and $\gamma$ are negative temperature coefficients and, consequently, the value of $S_{\mathrm{T}}$ resulting from (10) will be also negative. According to (7), higher values of $\mid \chi$ (and, hence, of $\left|S_{\mathrm{T}}\right|$ ) can be achieved by operating deeper in weak inversion, i.e. higher values of $W / L$ and/or lower values of $I_{B}$; these recommendations are opposite to those obtained in strong inversion [10]. Table II reports, for different MOSFETs biased with $I_{\mathrm{B}}=10 \mathrm{nA}$, the theoretical value of $S_{\mathrm{T}}$ calculated by (10) using the technology information provided in Table I and assuming $\eta=1.5$ [22]. Note that the values of $S_{\mathrm{T}}$ in weak inversion are not expected to be as high as those obtained in strong inversion [10] for a similar layout area occupied by the sensor.

The spread (or variability) of $S_{\mathrm{T}}$ (i.e. $\Delta S_{\mathrm{T}}$ ) due to IC manufacturing process variations can be evaluated by applying the law of propagation of uncertainties [23] in (10), thus resulting in:

$$
\Delta S_{\mathrm{T}}{ }^{2}=\left(\eta \frac{k}{q}\right)^{2}\left(\left(\frac{\Delta W}{W}\right)^{2}+\left(\frac{\Delta L}{L}\right)^{2}+\left(\frac{\Delta I_{\mathrm{B}}}{I_{\mathrm{B}}}\right)^{2}+\left(\frac{\Delta \mu_{0}}{\mu_{0}}\right)^{2}+\left(\frac{\Delta t_{\mathrm{OX}}}{t_{\mathrm{OX}}}\right)^{2}\right)+\Delta \beta^{2}
$$

where $\Delta W, \Delta L, \Delta I_{\mathrm{B}}, \Delta \mu_{0}, \Delta t_{\mathrm{OX}}$ and $\Delta \beta$ are, respectively, the spread (or variability) of $W, L, I_{\mathrm{B}}$, $\mu_{0}, t_{\mathrm{OX}}$ and $\beta$ due to process variations. As in [10], $\Delta S_{\mathrm{T}}$ depends on the relative variability of $W, L, I_{\mathrm{B}}, \mu_{0}$ and $t_{\mathrm{OX}}$, but here these are multiplied by $\eta k / q$, which is a small value $(129 \mu \mathrm{V} / \mathrm{K}$ for $\eta=1.5$ ). For example, if the quadratic sum of the relative variability of $W, L, I_{\mathrm{B}}, \mu_{0}$ and $t_{\mathrm{OX}}$ equals $10 \%$, then the resulting value of $\Delta S_{\mathrm{T}}$ equals $12.9 \mu \mathrm{V} / \mathrm{K}$, which is less than $1 \%$ of the nominal values of $S_{\mathrm{T}}$ reported in Table II. Accordingly, MOSFETs with a high $W$ and/or a high $L$ are not expected to carry major benefits in terms of spread of $S_{\mathrm{T}}$ when operating in 
weak inversion, although they do in strong inversion [10]. On the other hand, the term $\Delta \beta$ is usually neglected [24] but here its effects on $\Delta S_{\mathrm{T}}$ could be comparable to those discussed before.

As explained in Section II, the change of temperature $(\Delta T)$ to be detected by the MOSFET sensor shown in Fig. 1b is to be caused by a change of power $(\Delta P)$ dissipated by the CUT. Consequently, the change of the sensor output voltage $\left(\Delta V_{\mathrm{o}}\right)$ can be expressed as a function of $\Delta P$ as

$$
\Delta V_{\mathrm{o}}=S_{\mathrm{T}} \Delta T=S_{\mathrm{T}} R_{\mathrm{th}} \Delta P=S_{\mathrm{P}} \Delta P
$$

where $R_{\mathrm{th}}$ is a thermal coupling resistance that relates the change of temperature at the MOSFET sensor to the change of power dissipated by the CUT, and $S_{\mathrm{P}}$ is the sensitivity of the sensor to the power dissipated, which depends on both $S_{\mathrm{T}}$ and $R_{\mathrm{th}}$.

\section{SIMULATION RESULTS}

The temperature sensor shown in Fig. 1b has been simulated with Cadence in $0.35 \mu \mathrm{m}$ CMOS technology of AMS (AustriaMicroSystems) with $V_{\mathrm{DD}}=3.3 \mathrm{~V}$ and using BSIM3v3 models. Table II summarizes the dimensions of the MOSFETs simulated, which have a high $W / L$ so as to increase the sensitivity to temperature, as suggested by (7) and (10). The temperature dependence of such MOSFETs has been simulated through a DC temperaturesweep analysis assuming $300 \mathrm{~K}\left(27^{\circ} \mathrm{C}\right)$ as a reference (i.e. $\left.\Delta T=T-300 \mathrm{~K}\right)$.

Figure 2 shows how the output voltage of the sensor in Fig. 1b depends on temperature for different MOSFET dimensions when $I_{\mathrm{B}}=10 \mathrm{nA}$. For such dimensions, the output voltage decreases linearly with temperature, as predicted by (8). Furthermore, $S_{\mathrm{T}}$ increases (in absolute value) with the factor $W / L$, as expected from (7). MOSFETs M3 and M4, which have different values of $W$ and $L$ but the same $W / L$, show almost the same temperature dependence, as also expected from (7). The value of $S_{\mathrm{T}}$ of each of the cases represented in Fig. 2 and the corresponding value normalized to the maximum sensitivity (i.e. that of M3 or M4) are 
summarized in Table II. Note that the values of $S_{\mathrm{T}}$ resulting from simulations are very similar (differences are smaller than $10 \%$ ) to those calculated by (10) and, therefore, the models developed in Section III seem to be useful for a first estimation of $S_{\mathrm{T}}$.

Figure 3 shows how the output voltage of M3 depends on temperature for different bias currents $(5 \mathrm{nA}, 10 \mathrm{nA}$, and $20 \mathrm{nA})$. For the three currents simulated, the output voltage decreases linearly with temperature, as predicted by (8). In addition, as suggested by (7), $\left|S_{\mathrm{T}}\right|$ decreases with $I_{\mathrm{B}}$; to be precise: $S_{\mathrm{T}}$ equals $-1.602 \mathrm{mV} / \mathrm{K},-1.529 \mathrm{mV} / \mathrm{K}$ and $-1.455 \mathrm{mV} / \mathrm{K}$ for $5 \mathrm{nA}, 10 \mathrm{nA}$, and $20 \mathrm{nA}$, respectively. Note that decreasing $I_{\mathrm{B}}$ by a factor of 4 (e.g. from $20 \mathrm{nA}$ to $5 \mathrm{nA}$ ) or increasing $\mathrm{W} / \mathrm{L}$ by a factor of 4 (e.g. from M1 to M2, or from M2 to M3) brings about the same increase in $\left|S_{\mathrm{T}}\right|$ (around $150 \mu \mathrm{V} / \mathrm{K}$ in the previous examples).

The effects of process variations on $S_{\mathrm{T}}$ have been simulated through a Monte Carlo analysis of 50 runs. Assuming one standard deviation of $S_{\mathrm{T}}$ as $\Delta S_{\mathrm{T}}$, we have $\Delta S_{\mathrm{T}} \approx 10 \mu \mathrm{V} / \mathrm{K}$ for the different MOSFET dimensions simulated. Taking into account the values of $S_{\mathrm{T}}$ provided in Table II, the resulting $\Delta S_{\mathrm{T}} / S_{\mathrm{T}}$ is smaller than $1 \%$, which is quite similar to that obtained with BJTs [10]. Note, however, that the Monte Carlo analysis assumes the spread due to process variations of some parameters (e.g. $W, L, \mu_{0}, t_{\mathrm{OX}}$ and $V_{\mathrm{TH} 0}$ ) but not of others (such as $\alpha$ and $\beta$ ) and, therefore, the previous simulation results of $\Delta S_{\mathrm{T}} / S_{\mathrm{T}}$ could be too optimistic.

The sensor in Fig. 1b has also been simulated over the industrial temperature range (i.e. from $-40{ }^{\circ} \mathrm{C}$ to $85^{\circ} \mathrm{C}$ ) with the aim of evaluating how $S_{\mathrm{T}}$ depends on the operating temperature (note that in Figs. 2 and 3, it is assumed to be $27{ }^{\circ} \mathrm{C}$ ). The relative change of $S_{\mathrm{T}}$ (calculated as $\left.\left|\left(S_{\mathrm{T}}(T)-S_{\mathrm{T}}\left(27^{\circ} \mathrm{C}\right)\right) / S_{\mathrm{T}}\left(27^{\circ} \mathrm{C}\right)\right|\right)$ versus the operating temperature for different MOSFETs biased with $I_{\mathrm{B}}=10 \mathrm{nA}$ is represented in Fig. 4. Such simulations show that $S_{\mathrm{T}}$ undergoes a very small change (e.g. the change is smaller than $1 \%$ for M2, M3 and M4) and, therefore, the linear thermal response modeled by (8) seems to be applicable in the industrial 
temperature range. In comparison with the performance in strong inversion [10], MOSFETs in weak inversion offer a value of $S_{\mathrm{T}}$ that is more independent (up to ten times more) of the operating temperature and, consequently, they offer more linearity. Simulations have also shown that the effects of leakage currents coming from reverse-biased pn junctions (such as the drain-bulk junction) on the temperature dependence of the MOSFET are negligible, at least for temperatures lower than $85{ }^{\circ} \mathrm{C}$; for instance, the leakage current of the drain-bulk junction is around $10 \mathrm{pA}$ at $85^{\circ} \mathrm{C}$ for $\mathrm{M} 4$, which is a thousand times smaller than the bias current (i.e. $10 \mathrm{nA}$ ).

\section{EXPERIMENTAL RESULTS}

A chip including MOSFET-based temperature sensors with the topology shown in Fig. 1b and with different values of $W / L$ (those listed in Table II) has been implemented in $0.35 \mu \mathrm{m}$ 2P4M (2 levels of poly, 4 levels of metal) CMOS technology of AMS. The designed chip also includes heaters that are placed at $5 \mu \mathrm{m}$ from each of the sensors, as shown in Fig. 5a, with the purpose of testing the capability of the sensors to monitor on-chip thermal variations caused by the power dissipated by other embedded circuits; the device used as a heater is another diode-connected n-type MOSFET with $W_{\mathrm{h}}=450 \mu \mathrm{m}, L_{\mathrm{h}}=1 \mu \mathrm{m}$ and 15 fingers. Two additional blocks have been embedded into the chip (see Fig. 5b): (a) a current mirror that converts an external current $\left(I_{\mathrm{ext}}\right)$ from microampere to nanoampere range, and (b) an opampbased voltage follower that avoids the loading effects of external instrumentation. This opamp has (i) an input common mode voltage with a minimum value of $200 \mathrm{mV}$, which enables us to read the low voltage coming from the MOSFETs in weak inversion, and (ii) a maximum input offset voltage $\left(V_{\mathrm{IO}}\right)$ of $2 \mathrm{mV}$, whose effects are not expected to be critical here since we will represent the change of voltage with respect to that obtained at the beginning of the test (i.e. at $\Delta T=0$ or $\Delta P=0$ ). Furthermore, note that the MOSFET sensor is close to the heater (at $5 \mu \mathrm{m})$ and its sensitivity is around $1-2 \mathrm{mV} / \mathrm{K}$, whereas the opamp (and also the current mirror) is far 
from the heater (more than $200 \mu \mathrm{m}$ ) and the temperature coefficient of $V_{\mathrm{IO}}$ is much lower (typical values are $1-10 \mu \mathrm{V} / \mathrm{K}$ ). Consequently, the effects of the dissipated power on $V_{\text {IO }}$ (or other non-idealities of the opamp) are expected to be much lower than those on the sensor.

The temperature sensors have been initially tested by means of a heating chamber (MEMMERT, type UM-100), as shown in Fig. 5c; in this experiment, the embedded heaters did not dissipate power. A bench-top DC current source (Advantest R6240A) provided a stable $I_{\text {ext }}$ that was converted to the nanoampere range through the on-chip current mirror, whereas a digital multimeter (HP 34401A) measured the output $\left(V_{\mathrm{o}}\right)$ of the voltage follower. Moreover, the actual value of temperature inside the chamber was monitored by a reference temperature sensor (Pt100 IEC-751 Class A) whose value was measured by another digital multimeter (HP 3458A) applying the four-wire measurement method. Using this set-up, the output voltage of different MOSFETs biased with $I_{\mathrm{B}}=10 \mathrm{nA}$ was measured at different temperatures. The results are represented in Fig. 6, where the output voltage decreases linearly with temperature and, in addition, the higher the factor $W / L$, the higher the sensitivity, the same as in Fig. 2. If a straight line is fitted to the experimental data in Fig. 6 by means of the least-square method, the resulting maximum nonlinearity error (expressed as a percentage of the full-scale span, FSS) for the four MOSFETs is around $0.5 \% \mathrm{FSS}$, which is an acceptable value for IC testing purposes. The experimental values of $S_{\mathrm{T}}$ resulting from Fig. 6 and the corresponding value normalized to the maximum are reported in Table II. Note that the experimental values of $S_{\mathrm{T}}$ are very similar to those resulting from simulations (differences are smaller than $5 \%$ ). Experimental tests with the heating chamber also showed that the voltage follower was not able to correctly read the sensor output voltage at high temperatures (say, over $70^{\circ} \mathrm{C}$ ) because the voltage was smaller than $200 \mathrm{mV}$.

The temperature sensors have been then tested at room temperature to monitor on-chip thermal variations generated by the embedded heaters, as shown in Fig. $5 \mathrm{~d}$. The sensor was biased and measured using the same instrumentation indicated in Fig. 5c, whereas the heater 
was driven externally by a DC voltage source (Agilent E3631A); the current-voltage characteristic of the heater was previously acquired using R6240A and, therefore, the current (and, hence, the power) applied to the heater was accurately known for any voltage supplied later by E3631A. Using this set-up, the steady-state output voltage of the sensors was measured for different values of DC power applied to the heater and, then, $S_{\mathrm{P}}$ was calculated.

The effects of the power dissipated by the heater on the sensor output voltage are represented in Fig. 7 for different MOSFETs biased with $I_{\mathrm{B}}=10 \mathrm{nA}$, and in Fig. 8 for different bias currents applied to M3. In both figures, the output voltage decreases linearly with the dissipated power, as expected. Comparing the simulation results in Fig. 2 with the experimental results in Fig. 7, we realize that in both cases the sensitivity increases with $W / L$, but such an increase is smaller in Fig. 7. This discrepancy can also be observed by contrasting the normalized values of $S_{\mathrm{P}}$ resulting from Fig. 7 with those of $S_{\mathrm{T}}$ resulting from Fig. 2, as reported in Table II. Maybe the effective temperature change detected by the sensor was smaller than that expected for MOSFETs with a high $W$ (i.e. when the dimensions of the sensor were comparable to those of the heater) and, for this reason, there are such differences between Figs. 2 and 7. On the other hand, comparing the simulation results in Fig. 3 with the experimental results in Fig. 8, we can see that the effects of bias current on $S_{\mathrm{T}}$ and $S_{\mathrm{P}}$ are very similar. In both figures, the sensitivity increases with the same factor as the bias current decreases.

The spread of $S_{\mathrm{P}}$ due to process variations has also been experimentally evaluated by testing nine samples of the designed chip from the same batch. For example, $\Delta S_{\mathrm{P}} / S_{\mathrm{P}}$ was $1.2 \%$ for M3 using $I_{\mathrm{B}}=10 \mathrm{nA} ; \Delta S_{\mathrm{P}}$ is assumed to be one standard deviation of $S_{\mathrm{P}}$, in such a case: $20 \mu \mathrm{V} / \mathrm{mW}$ over $1.72 \mathrm{mV} / \mathrm{mW}$. Such an experimental value of $\Delta S_{\mathrm{P}} / S_{\mathrm{P}}$ is slightly higher than the simulated value of $\Delta S_{\mathrm{T}} / S_{\mathrm{T}}$ presented in Section IV and, consequently, the limitations of the Monte Carlo analysis indicated before do not seem so critical for a first estimation of 
the spread. In comparison with the experimental results reported in [10], MOSFETs operating in weak inversion offer values of $\Delta S_{\mathrm{P}} / S_{\mathrm{P}}$ very similar to those obtained with BJTs.

\section{DISCUSSION AND CONCLUSIONS}

The work presented herein together with that in [10] enable us to compare the performance of MOSFETs in weak inversion with that in strong inversion and also with that of parasitic (vertical) BJTs, always in the context of on-chip thermal testing. As summarized in Table III, several topics can be compared:

(a) CMOS compatibility: MOSFETs (either in strong or in weak inversion) are fully CMOS compatible and, therefore, they are preferable for the on-chip thermal testing of ICs fabricated in a CMOS process.

(b) Area: In CMOS technology, the use of parasitic BJTs is not as flexible as that of MOSFETs in terms of area. The well-characterized parasitic BJTs available in the design kits generally occupy a layout area that is much larger (say, ten times more) than that required using MOSFETs (either in strong or in weak inversion); for instance, the emitter area is $10 \times 10 \mu \mathrm{m}^{2}$ in $0.35 \mu \mathrm{m}$ from AMS, $10 \times 20 \mu \mathrm{m}^{2}$ in [9], and $15 \times 15 \mu \mathrm{m}^{2}$ in [25]. For the application described in Section II, a parasitic BJT with a smaller emitter area could be valid, but no reliable simulations could be carried out because of the lack of models; a preliminary tape-out intended for the experimental characterization of those small parasitic BJTs could solve such a limitation but at the expense of a higher cost. Note that this discussion about area mainly focuses on that occupied by the sensing transistor (and not that of the bias circuit or the BPF) since it is the most critical for the testing application of interest: the sensing transistor must be small enough to fit within the empty areas of the layout of the CUT with minimum impact on its design. 
(c) Sign of $S_{\mathrm{T}}$ : The output voltage of both MOSFETs in weak inversion and BJTs has a negative temperature coefficient, whereas that of MOSFETs in strong inversion usually shows a positive temperature coefficient.

(d) Magnitude of $\left|S_{\mathrm{T}}\right|$ : Using the sensitivity to temperature of BJTs as a reference, the sensitivity of MOSFETs in weak inversion is lower (around 20-30\% lower), whereas that in strong inversion can be significantly higher (more than three times higher).

(e) Advices to increase $\left|S_{T}\right|$ : The guidelines to increase the sensitivity to temperature in weak inversion (i.e. high $W / L$ and low bias current) are opposite to those obtained in strong inversion (i.e. low $W / L$ and high bias current). In terms of output noise voltage and output resistance, it is preferable to increase $\left|S_{T}\right|$ by using a higher value of $W / L$ in weak inversion and a higher value of the bias current in strong inversion. The fact of using a lower value of the bias current in weak inversion or a lower value of $W / L$ in strong inversion not only increases $\left|S_{\mathrm{T}}\right|$, but also the output noise voltage (to be precise, the white noise component) and the output resistance and, therefore, the circuit in Fig. $1 \mathrm{~b}$ becomes more susceptible to interference and the loading effects of the ensuing BPF (Fig. 1a) could be higher. As for the flicker noise component, the higher the factor $W \cdot L$, the smaller the output noise voltage [20], but the higher the area required around the CUT.

(f) Spread of $S_{\mathrm{T}}$ due to process variations: MOSFETs in weak inversion and BJTs have a similar susceptibility to process variations (i.e. $\Delta S_{\mathrm{T}} / S_{\mathrm{T}} \approx 1 \%$ ), which is around four times better than that obtained with MOSFETs in strong inversion. Note, however, that these results come from the experimental test of a reduced number of samples from the same batch and, therefore, higher values of spread could be expected if samples from different production batches were tested. 
(g) Linearity: In the industrial temperature range, MOSFETs in weak inversion offer a higher linearity (up to ten times more) and, therefore, the change of the output signal is expected to be almost the same regardless of the operating temperature.

(h) Current consumption: The required bias current is in the range of nanoamperes for MOSFETs in weak inversion, nanoamperes or microamperes for parasitic BJTs [9], and microamperes for MOSFETs in strong inversion [10]. If the opamp needed for the BPF in Fig. 1a has a current consumption of tens of microamperes, then the bias current of the MOSFET sensor becomes negligible in weak inversion but not in strong inversion and, consequently, the overall current consumption of the temperature sensor circuit is expected to be higher in strong inversion; note that the current consumption of the bias circuit is not assumed before because the temperature sensor circuit could easily make a good use of the bias circuit of the CUT.

According to the previous discussion, the major benefits of using MOSFETs in weak inversion in comparison with BJTs are: higher CMOS compatibility and lower layout area around the CUT. On the other hand, in comparison with MOSFETs in strong inversion, MOSFETs in weak inversion offer the following advantages: lower spread of $S_{\mathrm{T}}$ due to process variations (but always taking into account that the study here has been limited to a reduced number of samples from the same batch) and lower current consumption; this is, however, at the expense of a lower value of $S_{\mathrm{T}}$. The CUT to be tested applying the technique explained in Section II will also play a significant role in the selection of the most appropriate sensing transistor. For instance, for the on-chip thermal testing of a CUT that generates low changes of temperature, a MOSFET in strong inversion seems more appropriate because it offers higher values of $S_{\mathrm{T}}$. On the other hand, for a CUT that causes significant changes of temperature, a MOSFET in weak inversion could be more attractive since it enables us to optimize the testing sensor circuit in terms of power consumption.

For CMOS technologies different than that tested herein (i.e. $0.35 \mu \mathrm{m}$ from AMS), we can 
expect results similar to those shown in Table II provided that their technology parameters are similar to those presented in Table I; this has been proved through simulations carried out in $0.18 \mu \mathrm{m}$ CMOS technology from United Microelectronic Corporation (UMC). For technologies whose parameters are not so similar to those in Table I, the numerical results in Table II are not valid, but the qualitative conclusions given in Table III (for instance, advices to increase the sensitivity) are applicable; this has been proved through simulations performed in a $65 \mathrm{~nm}$ CMOS technology. Regardless of the technology being used, it is not recommended to design a MOSFET temperature sensor with the smallest value of $L$ or $W$, as usually happens in analog IC design, since it could increase both the output noise voltage and the spread of the sensitivity to temperature, as suggested by (11).

\section{ACKNOWLEDGMENTS}

This work has been funded by EU-Feder and Spanish MICINN under the TEC2008-01856

project and by AGAUR SGR 1497 funds. Authors highly appreciate the technical support of Enrique Barajas in the design of the chip layout. 


\section{REFERENCES}

[1] M. Sasaki, M. Ikeda, and K. Asada, "A temperature sensor with an inaccuracy of $-1 /+0.8$ ${ }^{\circ} \mathrm{C}$ using 90-nm 1-V CMOS for online thermal monitoring of VLSI circuits," IEEE Trans. Semicond. Manufacturing, vol. 21, no. 2, pp. 201-208, May 2008.

[2] P. Ituero, J.L. Ayala, and M. López-Vallejo, “A nanowatt smart temperature sensor for dynamic thermal management," IEEE Sensors Journal, vol. 8, no. 12, pp. 2036-2043, Dec. 2008.

[3] J. Altet, D. Gomez, X. Perpinyà, D. Mateo, J.L. González, M. Vellvehí, and X. Jordà, "Efficiency determination of RF linear power amplifiers by steady-state temperature monitoring using built-in sensors," Sens. Actuators A, Phys., vol. 192, pp. 49-57, 2013.

[4] E. Aldrete-Vidrio, D. Mateo, J. Altet, M. Amine Salhi, S. Grauby, S. Dilhaire, M. Onabajo, and J. Silva-Martinez, "Strategies for built-in characterization testing and performance monitoring of analog RF circuits with temperature measurements," Meas. Sci. Technol., vol. 21, pp. 075104 (10pp), 2010.

[5] Q. Yin, W.R. Eisenstadt, R.M. Fox, and T. Zhang, “A translinear RMS detector for embedded test of RF ICs," IEEE Trans. Instrum. Meas., vol. 54, no. 5, pp. 1708-1714, Oct. 2005.

[6] A. Valdes-Garcia, R. Venkatasubramanian, J. Silva-Martinez, and E. Sánchez-Sinencio, “A broadband CMOS amplitude detector for on-chip RF measurements," IEEE Trans. Instrum. Meas., vol. 57, no. 7, pp. 1470-1477, July 2008.

[7] C. Li, F. Gong, and P. Wang, "A low-power ultrawideband CMOS power detector with an embedded amplifier," IEEE Trans. Instrum. Meas., vol. 59, no. 12, pp. 3270-3278, Dec. 2010.

[8] F. Reverter, D. Gómez, and J. Altet, “On-chip MOSFET temperature sensor for electrical characterization of RF circuits," IEEE Sensors Journal, vol. 13, no. 9, pp. 3343-3344, Sept. 2013. 
[9] G. Wang and G.C.M. Meijer, "The temperature characteristics of bipolar transistors fabricated in CMOS technology," Sens. Actuators A, Phys., vol. 87, pp. 81-89, 2000.

[10] F. Reverter and J. Altet, "MOSFET temperature sensors for on-chip thermal testing," Sens. Actuators A, Phys., vol. 203, pp. 234-240, 2013.

[11] A. Kölling, F. Bak, P. Bergveld, and E. Seevinck, "Design of a CMOS temperature sensor with current output," Sens. Actuators, vol. A21-A23, pp. 645-649, 1990.

[12] K. Ueno, T. Asai, and Y. Amemiya, "Low-power temperature-to-frequency converter consisting of subthreshold CMOS circuits for integrated smart temperature sensors," Sens. Actuators A, Phys., vol. 165, pp. 132-137, 2011.

[13] D. Gómez, C. Dufis, J. Altet, D. Mateo, and J. L. González, “Electro-thermal coupling analysis methodology for RF circuits," Microelectronics J., vol. 43, pp. 633-641, 2012.

[14] J. Kuntner, F. Kohl, and B. Jakoby, "Simultaneous thermal conductivity and diffusivity sensing in liquids using a micromachined device," Sens. Actuators A, Phys., vol. 130-131, pp. 62-67, 2006.

[15] D. F. Reyes-Romero, O. Behrmann, G. Dame, and G. A. Urban, "Dynamic thermal sensor for biofilm monitoring," Sens. Actuators A, Phys., vol. 213, pp. 43-51, 2014.

[16] A. Wang, B. Highsmith, and A.P. Chandrakasan, Sub-threshold Design for Ultra LowPower Systems, New York: Springer, 2006.

[17] E. Vittoz and J. Fellrath, "CMOS analog integrated circuits based on weak inversion operation," IEEE Journal of Solid-State Circuits, vol. 12, no. 3, pp. 224-231, June 1977.

[18] Y.P. Tsividis, Operation and Modelling of the MOS Transistor, New York: McGrawHill, 1987.

[19] $0.35 \mu \mathrm{m}$ CMOS C35 Process Parameters, AustriaMicroSystems, 2008.

[20] B. Razavi, Design of Analog CMOS Integrated Circuits, New York: McGraw-Hill, 2001.

[21] V. Székely, M. Rencz, S.Török, Cs. Márta, and L. Lipták-Fegó, “CMOS temperature 
sensors and built-in test circuitry for thermal testing of ICs," Sens. Actuators A, Phys., vol. 71, pp. 10-18, 1998.

[22] R. Sarpeshkar, Ultra Low Power Bioelectronics, New York: Cambridge University Press, 2010.

[23] ISO 1993, Guide to the Expression of Uncertainty in Measurement, Geneva: International Organization for Standardization, corrected and reprinted 1995.

[24] I.M. Filanovsky and A. Allam, "Mutual compensation of mobility and threshold voltage temperature effects with applications in CMOS circuits," IEEE Trans. Circuits and Systems-I, vol. 48, no. 7, pp. 876-884, July 2001.

[25] M.A.P. Pertijs, G.C.M. Meijer, and J.H. Huijsing, "Precision temperature measurement using CMOS substrate PNP transistors," IEEE Sensors Journal, vol. 40, no. 3, pp. 294-300, June 2004. 


\section{SHORT BIOGRAPHIES}

Ferran REVERTER was born in Llagostera, Spain, on January 4, 1976. He received the B.Sc. degree in Industrial Electronic Engineering from the University of Girona (Girona, Spain) in 1998, the M.Sc. degree in Electronic Engineering from the University of Barcelona (Barcelona, Spain) in 2001, and the Ph.D. degree in Electronic Engineering from the Universitat Politècnica de Catalunya (UPC) (Barcelona, Spain) in 2004. Since 2001 he has been with the UPC, where he is an Associate Professor in Analogue Electronics and Digital Systems. He was a visiting postdoctoral researcher with the Delft University of Technology (Delft, The Netherlands) from 2005 to 2007, and with the Imperial College London (London, UK) in 2012. He is coauthor of the book Direct sensor-to-microcontroller interface circuits (Barcelona: Marcombo, 2005). His research interests are in the field of electronic instrumentation, in particular, the design of interface circuits for smart sensors.

Josep ALTET received the Engineering degree from the La Universitat Ramon Llull, La Salle and the Ph.D. degree from the Universitat Politècnica de Catalunya, UPC, Barcelona. He completed postdoctoral stays at The University of British Columbia, Université Bordeaux I, Centre Nacional de Microelectrònica, and Texas A\&M University. He is currently in the Department of Electronic Engineering, UPC, as Associate Professor. His research interests include VLSI design and test, temperature sensor design, and thermal coupling analysis and modeling in integrated circuits with applications to test and characterization of ICs. 


\section{List of Figure Captions}

Figure 1. (a) Sensor circuit for the measurement of on-chip low-frequency temperature signals resulting from the heterodyne technique. (b) Diode-connected MOSFET used as a temperature sensor $\left(\mathrm{T}_{1}\right)$ in Fig. 1a.

Figure 2. Simulated output-voltage change versus temperature for different MOSFETs biased with $I_{\mathrm{B}}=10 \mathrm{nA}$.

Figure 3. Simulated output-voltage change of M3 versus temperature for different bias currents.

Figure 4. Simulated relative change of $S_{\mathrm{T}}$ versus the operating temperature for different MOSFETs biased with $I_{\mathrm{B}}=10 \mathrm{nA}$.

Figure 5. (a) Sketch of the layout of the designed chip showing a heater-sensor pair. (b) Schematic of the electronics embedded into the designed chip. (c) Set-up to test the response of the sensors to temperature changes inside a heating camber. (d) Set-up to test the response of the sensors to on-chip thermal variations caused by the power dissipated by the embedded heater.

Figure 6. Experimental output voltage versus temperature for different MOSFETs biased with $I_{\mathrm{B}}=10 \mathrm{nA}$. The horizontal axis represents the increase of temperature $(\Delta T)$ applied through the heating chamber, whereas the vertical axis represents the change of voltage $\left(\Delta V_{\mathrm{o}}\right)$ with respect to that obtained at $\Delta T=0$.

Figure 7. Experimental output voltage versus power for different MOSFETs biased with $I_{\mathrm{B}}=10 \mathrm{nA}$. The horizontal axis represents the increase of power $(\Delta P)$ dissipated by the heater, whereas the vertical axis represents the change of voltage $\left(\Delta V_{\mathrm{o}}\right)$ with respect to that obtained at $\Delta P=0$.

Figure 8. Experimental output voltage of M3 versus power for different bias currents. The horizontal axis represents the increase of power $(\Delta P)$ dissipated by the heater, whereas the 
vertical axis represents the change of voltage $\left(\Delta V_{\mathrm{o}}\right)$ with respect to that obtained at $\Delta P=0$.

\section{List of Table Captions}

Table I. Main technology parameters for an n-type MOSFET in $0.35 \mu \mathrm{m}$ CMOS technology of AMS.

Table II. Theoretical, simulated and experimental values of sensitivity to temperature $\left(S_{\mathrm{T}}\right)$, and experimental values of sensitivity to power $\left(S_{\mathrm{P}}\right)$ for different MOSFETs biased with $I_{\mathrm{B}}=10 \mathrm{nA}$.

Table III. Comparative analysis between BJT- and MOSFET-based temperature sensors operating in either strong or weak inversion. 
Figure 1

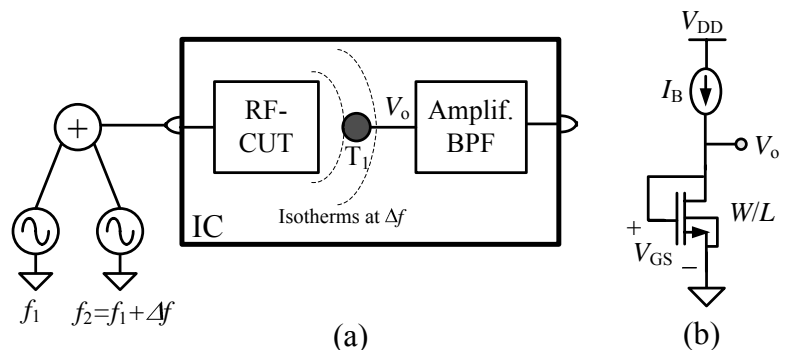

Figure 2

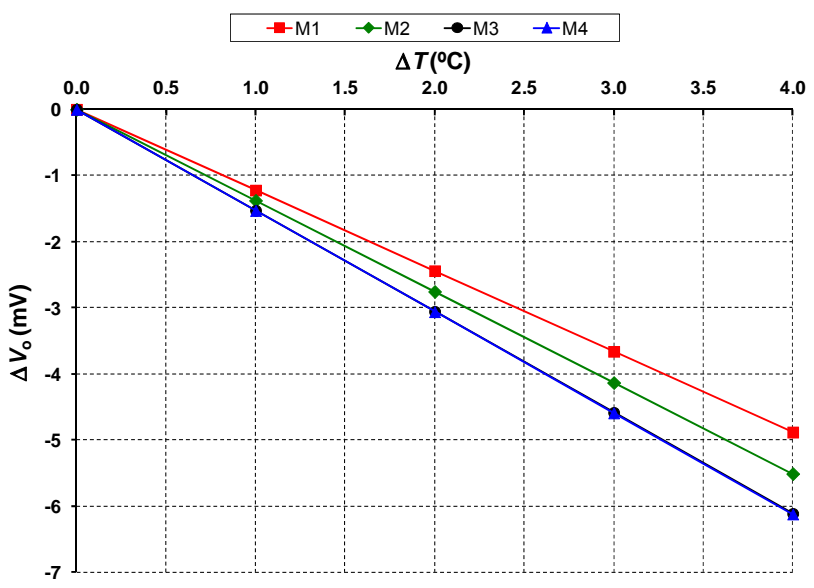

Figure 3

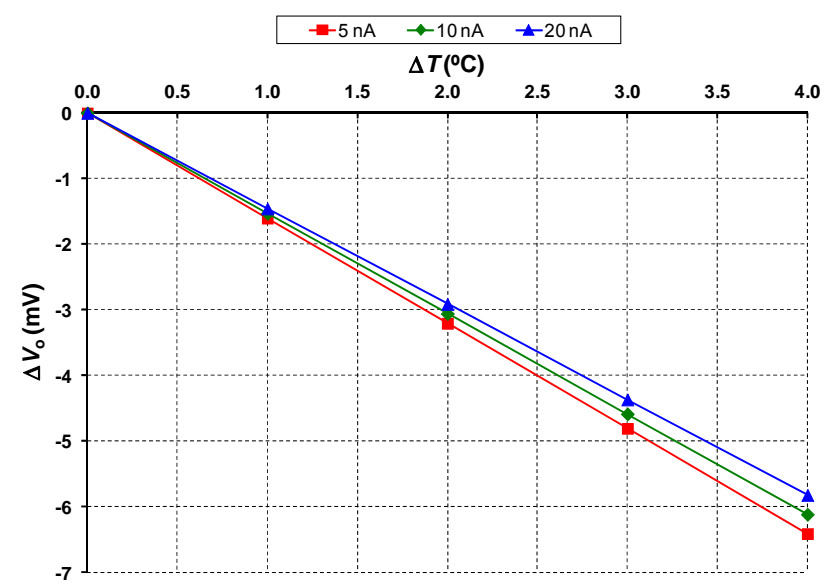


Figure 4

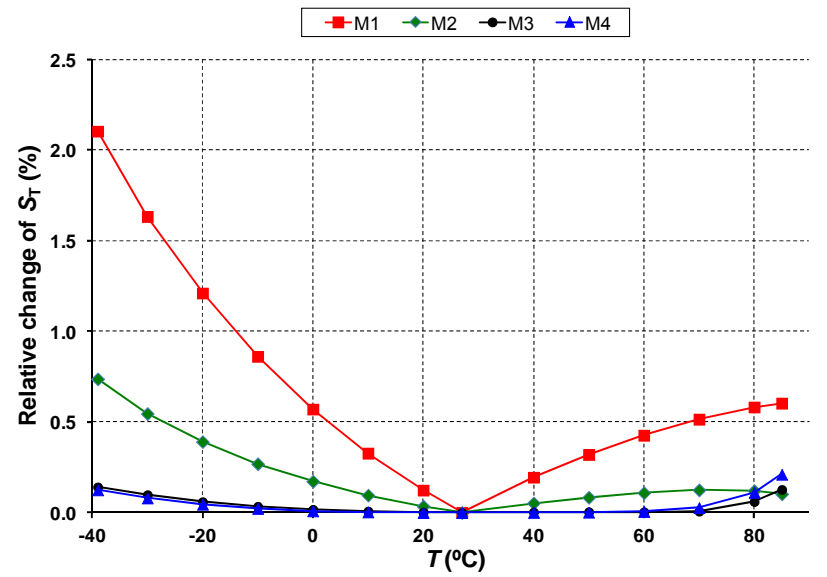

Figure 5

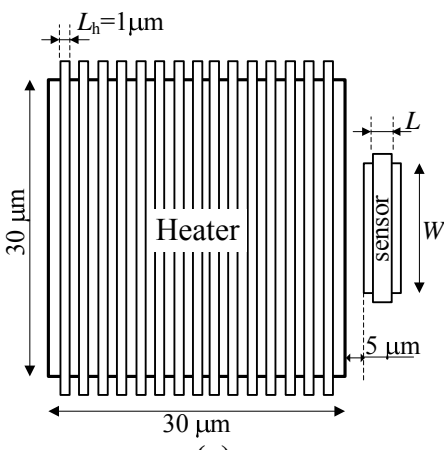

(a)

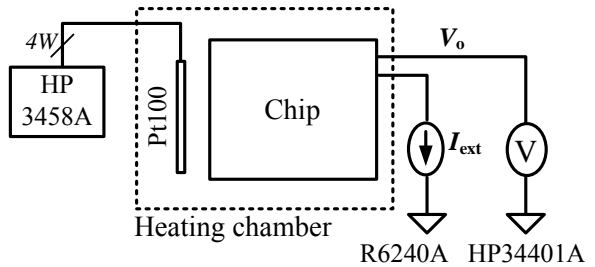

(c)

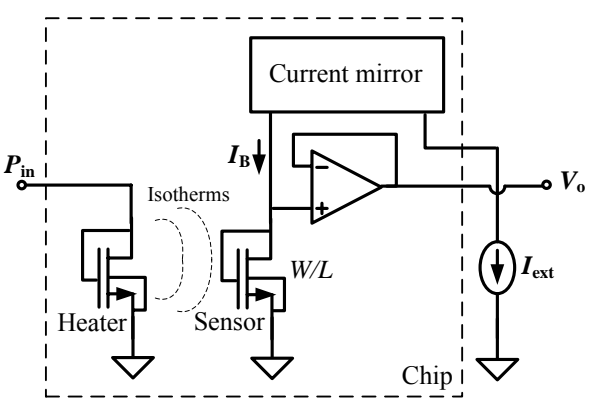

(b)

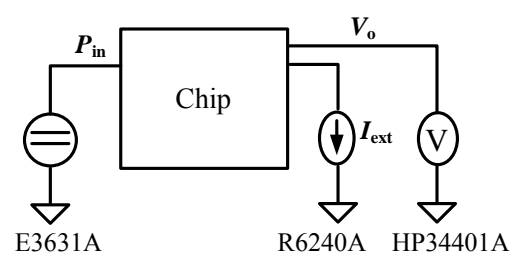

(d) 
Figure 6

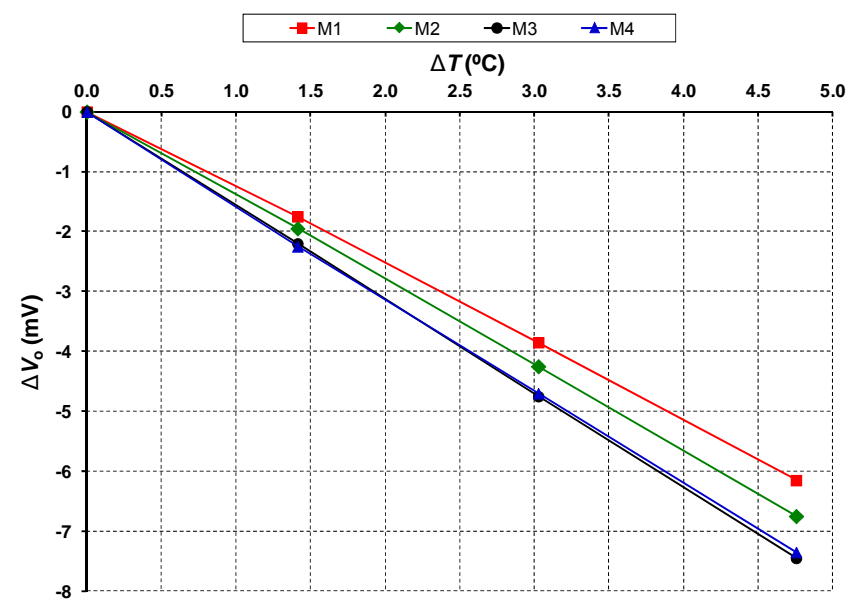

Figure 7

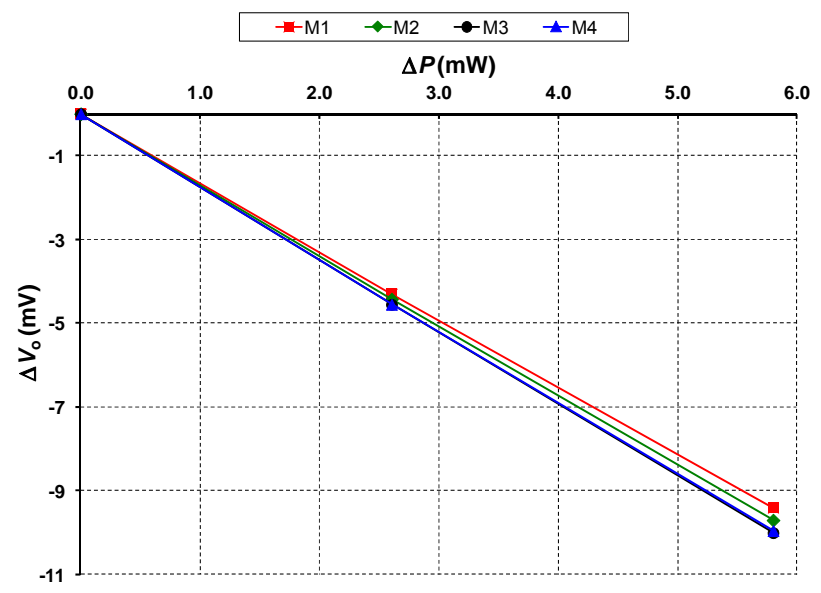

Figure 8

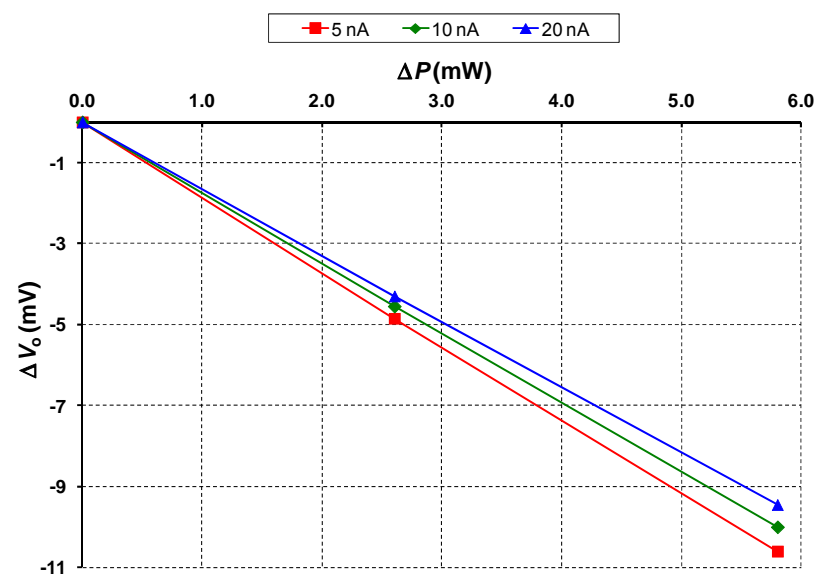


Table I

\begin{tabular}{|c|c|}
\hline Parameter & Value \\
\hline$\mu_{0}$ & $370 \mathrm{~cm}^{2} / \mathrm{V} / \mathrm{s}$ \\
\hline$V_{\mathrm{TH} 0}$ & $0.4979 \mathrm{~V}$ \\
\hline$\alpha$ & -1.8 \\
\hline$\beta$ & $-1.1 \mathrm{mV} / \mathrm{K}$ \\
\hline$t_{\mathrm{OX}}$ & $7.6 \mathrm{~nm}$ \\
\hline
\end{tabular}

Table II

\begin{tabular}{|c|c|c|c|c|c|c|c|c|c|}
\hline \multirow{3}{*}{ Sensor } & \multirow{3}{*}{$\begin{array}{c}W \\
(\mu \mathrm{m})\end{array}$} & \multirow{3}{*}{$\begin{array}{c}L \\
(\mu \mathrm{m})\end{array}$} & \multirow{3}{*}{$\begin{array}{c}\text { Theoretical } \\
\text { results } \\
\begin{array}{c}S_{\mathrm{T}} \\
(\mathrm{mV} / \mathrm{K})\end{array}\end{array}$} & \multirow{2}{*}{\multicolumn{2}{|c|}{ Simulation results }} & \multicolumn{4}{|c|}{ Experimental results } \\
\hline & & & & & & \multicolumn{2}{|c|}{ Heating chamber } & \multicolumn{2}{|c|}{$\begin{array}{l}\text { Power applied to the } \\
\text { heater }\end{array}$} \\
\hline & & & & $\begin{array}{c}S_{\mathrm{T}} \\
(\mathrm{mV} / \mathrm{K})\end{array}$ & $\begin{array}{c}\text { Norm. } \\
S_{\mathrm{T}}\end{array}$ & $\begin{array}{c}S_{\mathrm{T}} \\
(\mathrm{mV} / \mathrm{K})\end{array}$ & $\begin{array}{c}\text { Norm. } \\
S_{\mathrm{T}}\end{array}$ & $\begin{array}{c}S_{\mathrm{P}} \\
(\mathrm{mV} / \mathrm{mW})\end{array}$ & $\begin{array}{c}\text { Norm. } \\
S_{\mathrm{P}}\end{array}$ \\
\hline M1 & 1 & 1 & -1.32 & -1.22 & 0.80 & -1.29 & 0.82 & -1.62 & 0.94 \\
\hline M2 & 4 & 1 & -1.50 & -1.38 & 0.90 & -1.42 & 0.90 & -1.67 & 0.97 \\
\hline M3 & 16 & 1 & -1.68 & -1.53 & 1 & -1.57 & 1 & -1.72 & 1 \\
\hline M4 & 24 & 1.5 & -1.68 & -1.53 & 1 & -1.54 & 0.98 & -1.72 & 1 \\
\hline
\end{tabular}

Table III

\begin{tabular}{|c|c|c|c|}
\hline & BJT & $\begin{array}{c}\text { MOSFET in } \\
\text { strong inversion }\end{array}$ & $\begin{array}{c}\text { MOSFET in } \\
\text { weak inversion }\end{array}$ \\
\hline CMOS compatibility & Low-Medium & High & High \\
\hline Area & High & Low & Low \\
\hline Sign of $S_{\mathrm{T}}$ & Negative & Positive & Negative \\
\hline Magnitude of $\left|S_{\mathrm{T}}\right|$ & Medium & Medium-High & Low-Medium \\
\hline Advices to increase $\left|S_{\mathrm{T}}\right|$ & -- & $(W / L) \downarrow$ and $I_{\mathrm{B}} \uparrow$ & $(W / L) \uparrow$ and $I_{\mathrm{B}} \downarrow$ \\
\hline Spread of $S_{\mathrm{T}}$ due to process & Low & Medium & Low \\
\hline Linearity in $[-40,85]^{\circ} \mathrm{C}$ & Medium-High & Medium-High & High \\
\hline Current consumption & Low-Medium & Medium & Low \\
\hline
\end{tabular}

